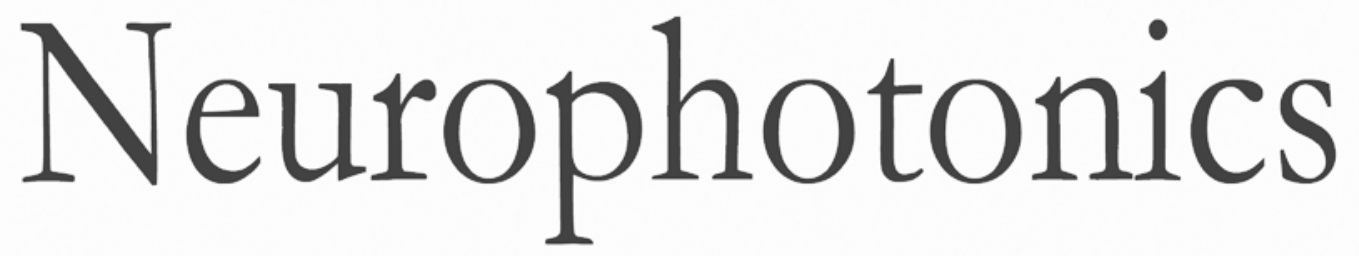

Neurophotonics.SPIEDigitalLibrary.org

\title{
Montreal SfNIRS conference shines light on the brain
}

Frédéric Lesage Hellmuth Obrig 


\title{
Montreal SfNIRS conference shines light on the brain
}

\author{
Frédéric Lesage \\ École Polytechnique Montréal \\ 2900 Edouard-Montpetit Boulevard \\ Montréal, Quebec H3T 1J4, Canada \\ and \\ Montréal Heart Institute \\ 5000 Rue Bélanger \\ Montréal, Quebec H1T 1C8, Canada \\ E-mail: frederic.lesage@polymtl.ca \\ Hellmuth Obrig \\ University Hospital Leipzig \\ Clinic for Cognitive Neurology \\ D-04103 Leipzig, Germany \\ and \\ Max-Planck-Institute for Human Cognitive and Brain Sciences \\ D-04103 Leipzig, Germany \\ E-mail: obrig@cbs.mpg.de
}

Noninvasive imaging of brain function has become an indispensable pillar of neuroscience and clinically motivated research on brain disease. Despite the undisputed strength of MRI-based techniques, less demanding and broadly affordable methods may be necessary to complement and expand the scope of neuroimaging from a bulky scanner to the bedside or into ecologically meaningful environments. Nearinfrared spectroscopy has clearly developed into one of the most exciting options increasingly used by cognitive and developmental researchers, neonatologists, anesthesiologists, neurologists, and psychiatrists. In concert with electrophysiological and other vascular-based imaging techniques, NIRS may well serve to link basic research to clinical challenges.

In October 2014, Montréal, Canada, welcomed the functional near-infrared spectroscopy conference fNIRS2014. From its inception in 2010 when it was held in Boston, United States, to its sequel in London, United Kingdom, in 2012, this biennial conference has seen increasing attendance and now attracts more than 300 international researchers working in fNIRS. It has become the main forum for presenting fNIRS research.

The wide range of contributions at the conference in Montréal reached from methodological advances, novel applications in cognitive and developmental neuroscience to clinical applications. While traditional NIRS imaging affords lowresolution images of limited areas of the brain's surface, the group from Washington University (Joe Culver) has steadily promoted a dense probe array setup allowing for a multidistance approach. This was shown to yield images with an impressive lateral spatial resolution approaching fMRI techniques. Such technological and analytical state-of-the-art setups can successfully image changes in neuronal connectivity in patients suffering from acute to subacute stroke.

These technologically demanding setups may be limited to use in hospitals and large research institutions. Thus it was exciting to see that the group from University College London

C 2015 Society of Photo-Optical Instrumentation Engineers (SPIE)
(Clare Elwell) has consistently followed the advantage of the instrumental portability of NIRS. Pioneering work on fNIRS imaging in global health issues, the group presented data on cognitive development in children in rural Africa. The data successfully collected in a village in Gambia allow for a first glimpse into the pressing questions of how malnutrition may interfere with normal cognitive development.

Beyond novel pioneering applications, the methodology may indeed provide broadly devisable diagnostic support. Martin Wolf (ETH Zürich) presented a multicenter phase II study on cerebral oxygenation monitoring in preterm infants, proving that NIRS can reach clinically meaningful usage. In adults beyond vascular disease, epilepsy has clearly become another focus of research. Altered hemodynamic responses may allow for better differentiation of seizure types. Focusing on epilepsy, two groups from Montreal (Dang Nguyen and Christophe Grova) have established the potential for longterm monitoring of patients. Especially when combined with EEG and MRI, fNIRS can refine the individualized multimodal search for an epileptic focus. Such dual assessment of the electrophysiological and the vascular response to pathological brain activity may be of utmost relevance in the immature brain. Fabrice Wallois (Amiens, France) convincingly showed how a novel and integrative view on vascular and electrophysiological responses to stimulation and pathological brain activity is mandatory when addressing neurodevelopment in infants and preterm babies. The range of applications in the many fields of developmental psychology is broad. Worldwide groups explore the methodology's potential in language acquisition and the development of other cognitive domains. Here, exciting new approaches based on dynamic connectivity analysis (Gentaro Taga, Tokyo, Japan) extend the spectrum. Other groups presented data on resting-state connectivity, a now widely adopted approach in the vascular imaging community to assess brain function and integrity of large-scale neuronal networks even without a defined stimulus protocol. This may well help to assess brain function also in populations with limited means to cooperate, such as elderly patients and children. 
The majority of fNIRS users rely on the basic parameters of hemoglobin oxygenation assessable by continuous-wave dual-wavelength systems. Less prominent signals including cell-based cytochrome-oxidase and the option to simultaneously measure complementary vascular parameters such as blood flow velocity require close cooperation between those groups with a focus on methodological advances with physiologists and clinicians. Thus the diversity of researchers and attendees from quite different fields shall be the path forward for accelerating these methodological advances into broadranging applications.

In that vein, the conference was combined with the creation of a new fNIRS society (fNIRS.org) with the goal of promoting exchange of ideas and research, creating standards, and encouraging communication within the community and with interdisciplinary partners. The society also promotes fNIRS through courses, activities, and dissemination of software. New board members and officers were elected in 2014, and president-elect Clare Elwell will continue to grow dissemination activities and help shape the future of the society in the coming years.
Exciting trends seen in fNIRS include an increasing use of fNIRS outside its mainstream founding applications (neonatal/ pediatrics, neurocognition) to applications in a clinical setting (stroke, epilepsy) and natural conditions (fNIRS in movement). Improvements in acquisition techniques such as broadband systems to measure cytochrome-c-oxidase, use of diffusion correlation spectroscopy to measure blood flow, gated time-domain detection toward improved brain-tissue sensitivity and increased optode density are all expected to help move the field further in next-generation acquisition consoles. fNIRS signal processing and data analysis also saw consolidation toward standards, which demonstrates the increased maturity of research in fNIRS.

In 2016, the fNIRS conference will be held in Paris (fNIRS2016), and we anticipate continued growth and scientific progress on the exciting topics and trends observed this year. Progress and innovations observed this year are expected to fuel scientific discoveries for years to come, and the next fNIRS conference will again be a central forum for dissemination and exchanges. We hope to see you all there. 